\title{
STUDY OF SIGNAL TRANSMISSION THROUGH DIFFERENT ARRAY OF Cu, Au AND Ag NANOSPHERES
}

\section{Dinesh Kumar Chaudhari and Bal Ram Ghimire}

Journal of Institute of Science and Technology

Volume 21, Issue 1, August 2016

ISSN: 2469-9062 (print), 2467-9240(e)

Editors:

Prof. Dr. Kumar Sapkota

Prof. Dr. Armila Rajbhandari

Assoc. Prof. Dr. Gopi Chandra Kaphle

JIST, 21 (1), 133-139 (2016)

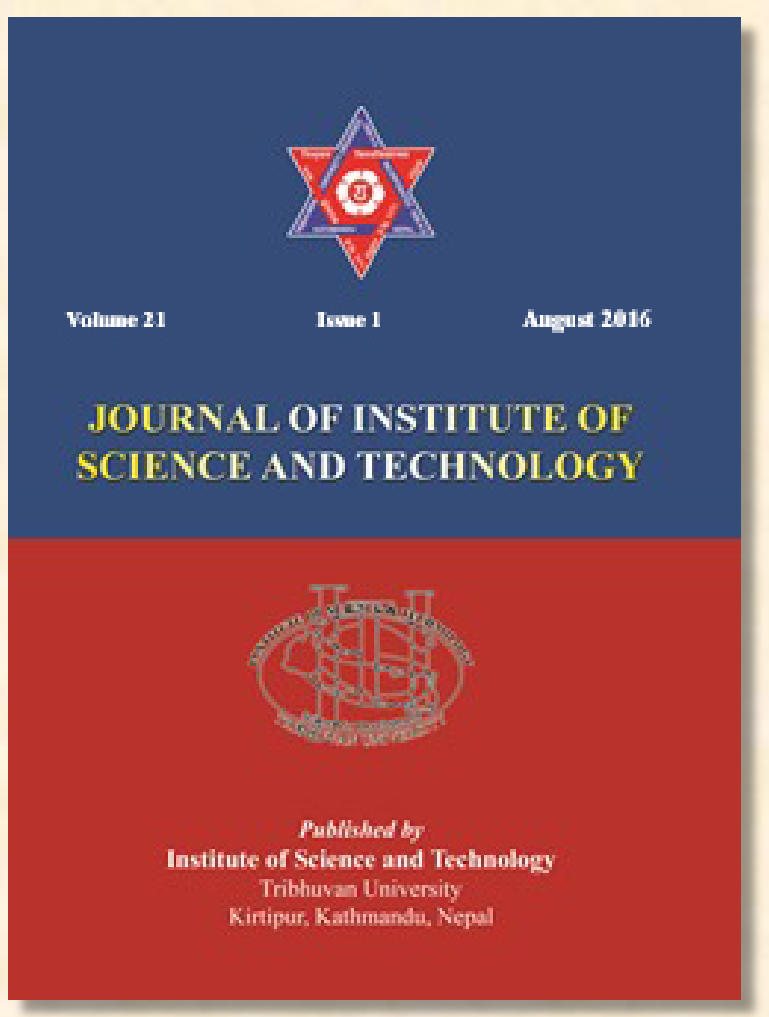

Published by:

Institute of Science and Technology

Tribhuvan University

Kirtipur, Kathmandu, Nepal 


\title{
STUDY OF SIGNAL TRANSMISSION THROUGH DIFFERENT ARRAY OF Cu, Au AND Ag NANOSPHERES
}

\author{
Dinesh Kumar Chaudhari ${ }^{1}$ and Bal Ram Ghimire ${ }^{1,2, *}$ \\ ${ }^{1}$ Central Department of Physics, Tribhuvan University, Kirtipur, Nepal \\ ${ }^{2}$ University of North Dakota, ND, USA \\ *Corresponding email: balramghimire@gmail.com
}

\begin{abstract}
Twenty first century is the age of Information Technology and we always look for faster information transport and processing capabilities. Data can be moved by transistors and optical fibers. Transistors carry small amount of data and are small in size while optical fibers can carry huge amount of data but are much bigger in size. Metal nanostructures may possess right combination of electric and optical properties to tackle the issues outlined above and realized dream of significantly faster processing speeds. In this work, dispersion relation in Plasmon modes in linear chain and alternated chain of nanoparticles like silver-gold, and gold-copper were studied. Expressions of angular frequency of Plasmon modes, group- velocity and extinction- coefficient were derived by solving equation of motion of Plasmon oscillation on the single kind of nanoparticle and that on different alternated chain of nanoparticles. Expressions of angular frequency of Plasmon modes, group velocity and extinction coefficient were tested for specific linear chain of nanoparticles. Calculation showed that extinction coefficients for the linear chain of Copper, Gold and Silver nanoparticles are less and group velocities higher than that of alternated silver-gold and alternated gold-copper at their respective resonance frequencies
\end{abstract}

Key Words: Localized surface plasmon polariton, Point dipole approximation, Surface plasmon

\section{INTRODUCTION}

Quantum of collective longitudinal excitation of conduction electron on the surface is called Surface Plasmon (Kittle 2004). This excitation of conduction electron on the metal surface can be achieved using beam of accelerated electron and electromagnetic (EM) radiation as well. In 1957, Refuse Ritchie (Ritchie 1957) published a finding on electron energy losses in thin films, in which it was shown that plasmon modes can exist near the surface of metals. It was the first theoretical description of surface plasmons. In 1970 Uwe Keribig and Peter Zacharias (Kreibig \& Zacharias 1970), performed a study in which they compared the electronic and optical responses of gold and silver nanoparticles. For the first time they described the optical properties of metal nanoparticles in terms of surface plasmon. In case of noble metal nanospheres, this exciting EM radiation lies in the range of the visible and nearinfrared energy range. Excited plasmon by the interaction of light radiation with electron cloud is called Plasmon Polariton (Barnes et al. 2003, Brongersma et al. 2007). At first glance, the use of metallic structures to transmit light signals seems impractical, because metals are known for high optical loss. But using simulation, it has been shown that electromagnetic radiation can be transmitted through the array of closely spaced metallic nanospheres (Quinten et al. 1998 \& Atwater 2007). Using the Silver nanospheres chain below the diffraction limit of visible range light, possibility of transportation and extinction of signal had been studied by the Brongersma et al. 2000 (Brongersma et al. 2000) using point dipole approximation. They showed that signal can be transmitted using such an array of metallic nanospheres even at the speed of $0.1 \mathrm{c}$.

At the early stage, information transporting devices used to be bulky and slow in performance. Discovery of semiconductor followed by the transistor greatly increased speed of propagation of signal and hence tuned new era in information transportation technology. In this process not only speed was greatly increased, as illustrated in Fig. 1, but also there was reduction in size of device using advance nanotechnology. These semiconductor and transistor based devices let us to design circuit that 


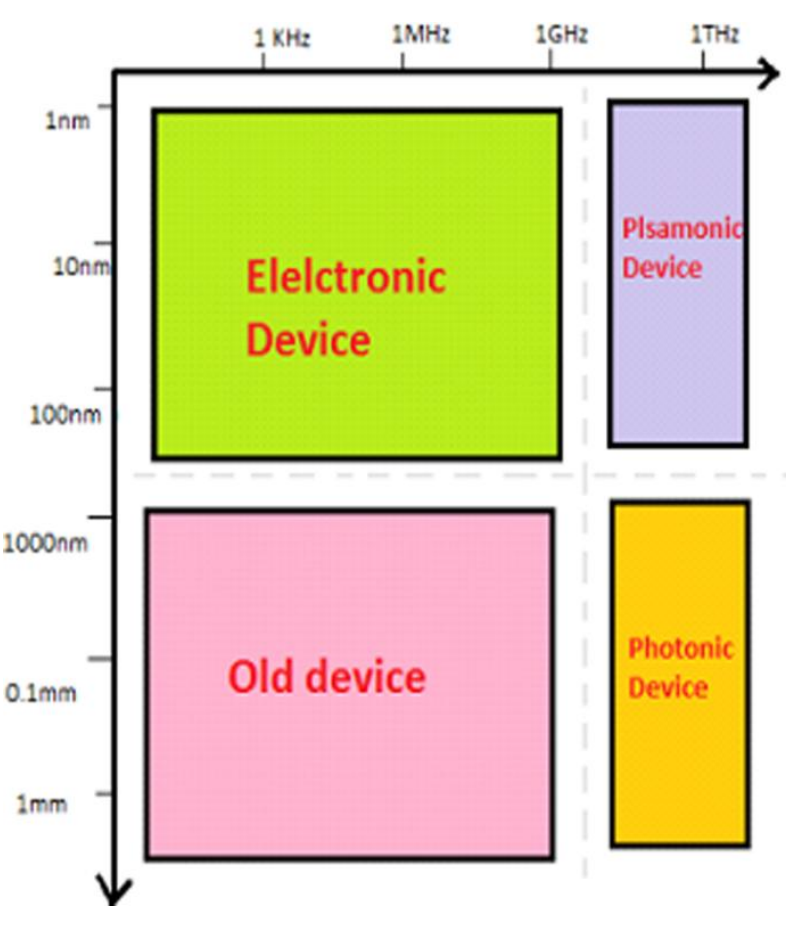

Fig. 1. Progress in signal transporting device speed and dimension.

can transport signal in terms of electric current. Nano-scale study of matter has allowed us to design transistor even within 50nm (Ozbay 2006). But their efficiency is bounded by the velocity of electron flow through the wire. Optical signal carrying devices, like optical fiber can carry signal with efficiency > 1000 times (Ozbay 2006), is desirable to find out structures that can interconnect optical signal carrying device and electric signal carrying bulky device (example: optical fibers that are bulky in size compared to that of present very tiny nanoscale level chips and circuit elements like transistor, capacitor, etc.). Metallic nanospheres can interact with electromagnetic radiation and excites (LSPP) on it that oscillates at the frequency many times greater that of incident radiation (Barnes et al. 2003). This compresses signal many times to very short wavelength range so that signal can pass through structure below its diffraction limit. Thus, the scarcity of efficient and fast interconnector can be fulfilled by the nanospheres array of metal, as nanospheres array of metal can provide guide to light signal by the mean of electron cloud oscillation on its surface, and hence allows signal to propagate through it. This permits us to design whole ultrafast signal processing circuit within a nano-scale range with ultrafast speed (Atwater 2007).

\section{MATERIALS AND METHODS}

This work tried to describe attenuation coefficients and signal propagation velocity, for signal that lies within visible and red infrared region, through array of linear and alternated nanospheres of Copper $(\mathrm{Cu})$, Gold $(\mathrm{Au})$ and Silver $(\mathrm{Ag})$ based on point dipole approximation. In this analytical method of investigation, we have used classical electrodynamics' concept that can deal with oscillating dipole and their interactions.

Under the incident monochromatic electromagnetic (EM) radiation of wavelength, $\lambda$, greater than that of radius, $R$, and inter-particle spacing between neighboring nanospheres, $d$, there are homogenous polarization of nanospheres in the array and get polarized long the direction of incident electric field giving Longitudinal mode (L-mode) and Transverse mode (T-mode) of polarization depending upon the polarization of nanopsheres along the axis and perpendicular to the axis of nanospheres array respectively. Electric field by an individual oscillating dipole $\boldsymbol{P}_{i, m, j}$, due to $j^{\text {th }}$ types of material located at $m^{\text {th }}$ position in $i^{\text {th }}$ mode of polarization, at the neighboring nanospheres of $k^{\text {th }}$ types of material located at $(m+1)$ and $(m-1)$ position, depends upon the polarization dependent constant $\gamma_{i}$, is given by an equation (Brongersma $e t$ al. 2000),

$$
\boldsymbol{E}_{i, j, m}=\frac{\gamma_{i} \boldsymbol{P}_{i, j, m}}{4 \pi \epsilon_{0} d^{3}} .
$$

Electric field is dependent of $d^{-3}$ and hence there is significance of near field rather than far field in coupling phenomena. Here we have used $\epsilon_{0}$ for dielectric constant of free space, $i=L$ and $i=T$ for $\mathrm{L}$-mode and $\mathrm{T}$-mode respectively such that $\gamma_{L}=-2$ and $\gamma_{T}=1$ considering $j, k=1,2,3$ for $\mathrm{Cu}, \mathrm{Au}$ and $\mathrm{Ag}$ respectively. Dipole on Localized Surface Plasmon (LSPP) at $j^{\text {th }}$ type of nanospherematerial oscillates with frequency $\omega_{0, j}$, can be obtained in terms of bulk plasma frequency $\omega_{p, j}$ of $j^{\text {th }}$ types of material given by Drude model of metal. Due to oscillation of dipole with Eigen frequency of oscillation, $\omega_{0, j}$, there is radiation loss and its affect can be expressed in term of relaxation frequency, $\Gamma_{R, j}=\omega_{0, j}^{2} \tau_{j}$, with $\tau_{j}=\frac{2 e^{2}}{3 m_{j}^{*} c^{3}}$ (Jackson 2013) such that $e, m_{j}^{*}$, and $c$ are representing electric charge, optical effective mass of an electron, and velocity of light respectively. Oscillating LSPP also suffers damping due to non radiative loss like electron hole formation, hot electron formation, and phonon excitation, that 
depends on the Fermi velocity, $V_{F, j}$, bulk mean free path, $\lambda_{B, j}$, and radius of nanosphere, $R$, and hence give heating effect. This gives relaxation frequency for $j^{\text {th }}$ type of material nanosphere, $\Gamma_{l, j}$, given by the relation (Kreibig \& Genzel 1985),

$\Gamma_{l, j}=\frac{V_{F, j}}{\lambda_{B, j}}+\frac{V_{F, j}}{R}$.

There is coupling between nearby nanospheres through the electric field interaction between oscillating dipoles, and hence give rise coupling frequency of $j^{\text {th }}$ types of nanospheres, $\omega_{1, j}$, depends upon the dipole charge, $q_{j}$, as well as center to center spacing between nanospheres, $d$, and optical effective mass of electron, $m_{j}^{*}$, as given by equation (Brongersma et al. 2000),

$\omega_{1, j}^{2}=\frac{q_{j} e}{4 \pi m_{j}^{*} \epsilon_{0} d^{3}}$.

With above consideration, equation of oscillation of nanospheres array, made by two different material of types $j$ and $k$, is given by the relation,

$\ddot{\boldsymbol{P}}_{i, j, m}=-\omega_{0, j}^{2} \boldsymbol{P}_{i, j, m}-\Gamma_{l, j} \dot{\boldsymbol{P}}_{i, j, m}+\frac{\Gamma_{R, j}}{\omega_{0,1}^{2}} \dddot{\boldsymbol{P}}_{i, j, m}$

$+\gamma_{i} \omega_{1, j}^{2}\left(\boldsymbol{P}_{i, k, m+1}+\boldsymbol{P}_{i, k, m-1}\right)$.

This is equation of damped harmonic oscillator giving resultant acceleration of LSPP, $j^{\text {th }}$ types of material, located at $m^{\text {th }}$ position. First term on right side is arising from the Eigen oscillation frequency of LSPP on nanosphere, considered at center in the array of nanospheres. Second and third terms give acceleration arising from non-radiative and radiative energy loss. The last term is giving effect of coupling between nanospheres, of $j^{\text {th }}$ type and $k^{t h}$ of materials, on the array. As this equation is representing damped harmonic oscillator, it is desirable to introduce solution of above equation in the form of,

$$
\boldsymbol{P}_{i, j, m}=\boldsymbol{P}_{i, j, 0} e^{i(\omega t-K m d)} e^{-\alpha_{i} m d},
$$

and

$\boldsymbol{P}_{i, k, m}=\boldsymbol{P}_{i, k, 0} e^{i(\omega t-K m d)} e^{-\alpha_{i} m d}$.

$\boldsymbol{P}_{i, j, 0}$ and $\boldsymbol{P}_{i, k, 0}$ are dipole moments of LSPPs on nanospheres of $j^{\text {th }}$ and $k^{\text {th }}$ type of material located as the first nanospheres being excited by the radiation respectively. Exponential terms with attenuation coefficient, $\alpha_{i}$, give damping on the oscillation and other exponentials without $\alpha_{i}$ give Plasmon oscillation without damping. Solving above equations we get real part and imaginary part. Real part gives dispersion equation,

$\omega^{2}=0.5\left(\omega_{0, j}^{2}+\omega_{0 . k}^{2}+\Gamma_{l, j} \Gamma_{l, k}\right)+0.5$ $\sqrt{\left(\omega_{o, j}^{2}-\omega_{0, k}^{2}\right)^{2}+16 \gamma_{i}^{2} \omega_{1, j}^{2} \omega_{1, k}^{2} \sin ^{2}(K d)}$.

Using the group velocity relation, $V_{g, i}=\frac{\partial \omega}{\partial K}$ and approximating $\cos h\left(\alpha_{i} d\right) \approx 1, \quad \sinh \left(\alpha_{\mathrm{i}} d\right) \approx$ $\left(\alpha_{i} d\right) \ll 1$ for very small damping, we get group velocity at $i^{\text {th }}$ mode of polarization,

$V_{g, i}=\left[4 \gamma_{i}^{2} d \omega_{1, j}^{2} \omega_{1, k}^{2} \sin (2 \mathrm{Kd})\right] /$

$\left\{\begin{array}{c}2 \omega\left(\omega_{0, j}^{2}+\omega_{0, k}^{2}+\Gamma_{l, j} \Gamma_{l, k}\right)-4 \omega^{3} \times \\ \left(1-\frac{\Gamma_{R, j} \Gamma_{l, k}}{\omega_{0, j}^{2}}+\frac{\Gamma_{R, k} \Gamma_{l, j}}{\omega_{0, k}^{2}}\right)\end{array}\right\}$.

Imaginary part of the solution gives attenuation coefficient, $\alpha_{i}$ gives the energy loss of signal on passing through the signal carrier, in terms of group velocity, damping frequencies, etc. as,

$$
\begin{aligned}
& \alpha_{i}= \\
& \left\{\begin{array}{c}
\omega^{3}\left\{\left(\frac{\Gamma_{R, k} \omega_{0, j}^{2}}{\omega_{0, k}^{2}}+\frac{\Gamma_{R, j} \omega_{0, k}^{2}}{\omega_{0, j}^{2}}\right)-\left(\Gamma_{l, j}+\Gamma_{l, k}\right)\right\} \\
+\omega\left\{\Gamma_{l, k} \omega_{0, j}^{2}+\Gamma_{l, j} \omega_{0, k}^{2}\right\}-\omega^{5}\left\{\frac{\Gamma_{R, j}}{\omega_{0, j}^{2}}+\frac{\Gamma_{R, k}}{\omega_{0, k}^{2}}\right\}
\end{array}\right\} \\
& \div\left[V _ { g , i } \left\{\begin{array}{c}
2 \omega\left(\omega_{0, j}^{2}+\omega_{0, k}^{2}+\Gamma_{l, j} \Gamma_{l, k}\right) \\
\left.-4 \omega^{3}\left(1-\left(\frac{\Gamma_{R, j} \Gamma_{l, k}}{\omega_{0, j}^{2}}+\frac{\Gamma_{R, k} \Gamma_{l, j}}{\omega_{0, k}^{2}}\right)\right)\right\} .
\end{array}\right.\right.
\end{aligned}
$$

\section{RESULTS AND DISCUSSION}

We have carried out calculations for the array of alternated as well as array of single type nanospheres, of radius $R=25 \mathrm{~nm}$, materials like $\mathrm{Cu}, \mathrm{Au}$ and $\mathrm{Ag}$, separated by distance $d=75 \mathrm{~nm}$. Used effective mass of electron in $\mathrm{Cu}, \mathrm{Au}$ and $\mathrm{Ag}$ are $m_{1}^{*}=1.49 m, m_{2}^{*}=0.99 m$ and $m_{3}^{*}=0.96 m$ respectively (Johnson \& Christy 1972), where $m=9.1 \times 10^{-31} \mathrm{Kg}$ is rest mass of electron. On using bulk electron densities for $\mathrm{Cu}, \mathrm{Au}$ and $\mathrm{Ag}$ as $8.45 \times 10^{28} \mathrm{~m}^{-3}, 5.90 \times 10^{28} \mathrm{~m}^{-3}$ and $5.85 \times$ $10^{28} \mathrm{~m}^{-3}$ respectively (Kittle 2004), we got their respective coupling frequencies, $\omega_{1,1}=1.49 \times$ $10^{15} \mathrm{rad} / \mathrm{s}, \omega_{1,2}=1.53 \times 10^{15} \mathrm{rad} / \mathrm{s}$ and $\omega_{1,3}=$ $1.54 \times 10^{15} \mathrm{rad} / \mathrm{s}$, and their individual nanosphere oscillation frequencies, $\omega_{0,1}=4.75 \times 10^{15} \mathrm{rad} / \mathrm{s}$ (Chen et al. 2011), $\omega_{0,2}=4.87 \times 10^{15} \mathrm{rad} / \mathrm{s}$ and $\omega_{0,3}=4.92 \times 10^{15} \mathrm{rad} / \mathrm{s}$. Here we see coupling frequencies and resonance oscillation frequencies of individual dipole for our considered systems are of same order and hence there are possibilities of strong coupling interactions. Fermi velocities of 
electron on $\mathrm{Cu}, \mathrm{Au}$ and $\mathrm{Ag}$ bulk materials, $V_{F, 1}=$ $1.57 \times 10^{6} \mathrm{~m} / \mathrm{s}, V_{F, 2}=1.39 \times 10^{6} \mathrm{~m} / \mathrm{s} \quad$ and $V_{F, 3}=1.38 \times 10^{6} \mathrm{~m} / \mathrm{s}$ (Kittle 2004) together with their respective bulk mean free paths, $\lambda_{B, 1}=39 \mathrm{~nm}$ (Sattler 2016), $\lambda_{B, 2}=41 \mathrm{~nm}$ (Waser 2012) and $\lambda_{B, 3}=57 \mathrm{~nm}$ (Brongersma et al. 2000) respectively. This results, their correspondence relaxation frequencies are $\Gamma_{l, 1}=10.30 \times$ $10^{13} \mathrm{rad} / \mathrm{s}, \quad \Gamma_{l, 2}=8.95 \times 10^{13} \mathrm{rad} / \mathrm{s}$ and $\Gamma_{l, 3}=$ $7.94 \times 10^{13} \mathrm{rad} / \mathrm{s}$ respectively. Characteristic time period of oscillation $\tau_{j}$ of electrons in $\mathrm{Cu}, \mathrm{Au}$ and $\mathrm{Ag}, 4.20 \times 10^{-24} \mathrm{~s}, 6.33 \times 10^{-24} \mathrm{~s}$ and $6.52 \times$ $10^{-24} \mathrm{~s}$ respectively, give their respective relaxation time frequencies due to radiation loss, $\Gamma_{R, 1}=2.53 \times 10^{8} \mathrm{rad} / \mathrm{s}, \Gamma_{R, 2}=4.00 \times 10^{8} \mathrm{rad} / \mathrm{s}$ and $\Gamma_{R, 3}=4.22 \times 10^{8} \mathrm{rad} / \mathrm{s}$ respectively. From here we get $\Gamma_{R, j} \ll \Gamma_{l, j}$, and can conclude that effect on damping of nanospheres due to radiation loss is negligible than that of non-radiative loss.

\section{(i) Dispersion Curve:}

On the basis of equation (6), dispersion equation for single type of nanospheres array of $\mathrm{Cu}, \mathrm{Au}$ and $\mathrm{Ag}$ is illustrated in Fig. 2. Here we see that, frequency of signal, propagating through the array, oscillates about certain central value. Allowed frequency region for $\mathrm{Cu}$ nanospheres array is slightly lower than that of $\mathrm{Au}$, and $\mathrm{Ag}$ has upper frequency region. Band width for the array of $\mathrm{Cu}$, array of $\mathrm{Au}$ and array of $\mathrm{Ag}$ nanospheres shows almost same magnitudes. Thus array of individual $\mathrm{Cu}, \mathrm{Au}$ and $\mathrm{Ag}$ nanospheres can carry nearly same volume of data although their efficiencies may be different. Dispersion curves for T-mode, denoted by dotted lines, has almost half band width of corresponding L-mode with same period of variation with wave vector $\boldsymbol{K}$. This signifies, Tmode excitation of LSPP on array of nanospheres cannot propagate large amount of data as much as that can be done by corresponding L-mode. This phenomenon also appears on the array of alternated $\mathrm{Cu}$ and $\mathrm{Au}$ nanospheres, and array of alternated $\mathrm{Au}$ and $\mathrm{Ag}$ nanospheres as shown in Fig. 3.

This phenomenon together with equation (7) of velocity shows that signal can propagate in L-mode almost two times faster than that of corresponding T-mode. Dispersion curves for array of alternated nanosperes made by $\mathrm{Cu}$ and $\mathrm{Au}$, and that for array of alternated nanospheres made by $\mathrm{Au}$ and $\mathrm{Ag}$ is shown in Fig. 3.

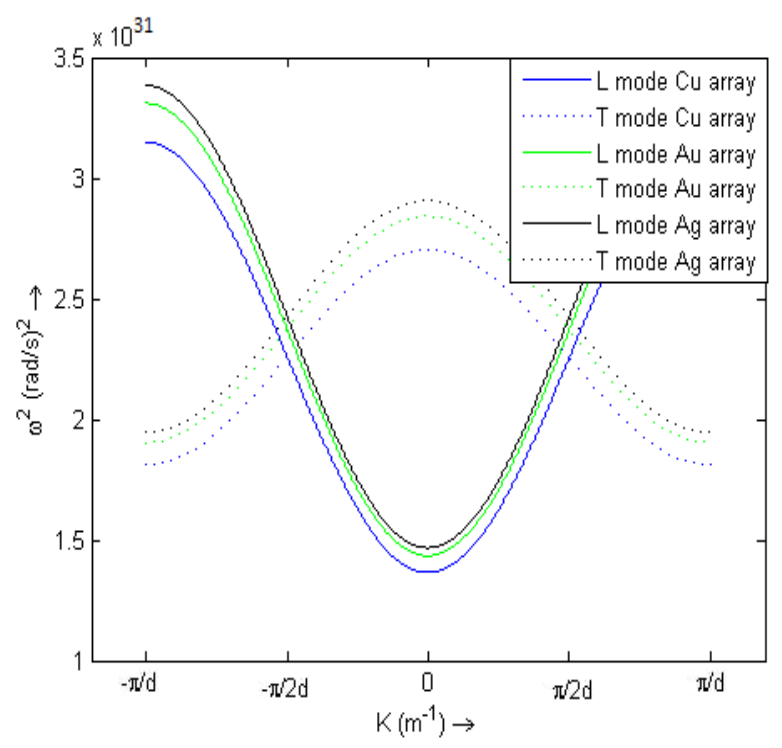

Fig. 2. Dispersion curve for array of nanospheres of (a) Cu, denoted by blue lines (b) Au, denoted by green lines and (c) Ag, denoted by black lines. Dotted lines are drawn for their respective $T$ modes and solid lines for L-modes.

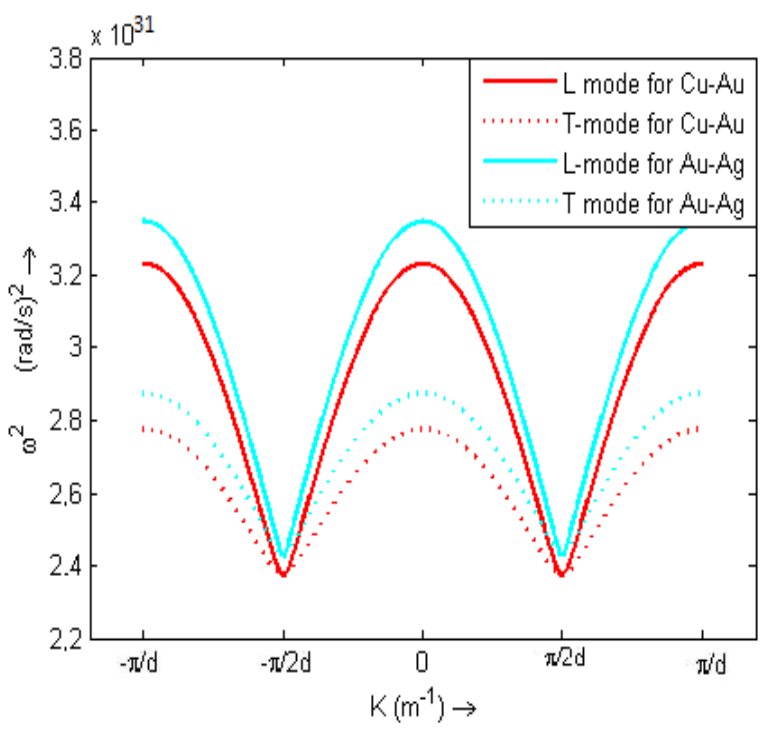

Fig. 3. Dispersion curve for alternated nanospheres array of (a) $\mathrm{Cu}$ and $\mathrm{Au}$ nanospheres, denoted by red color and (b) Au and Ag nanospheres, denoted by cyan colored lines. Here, dotted lines are representing their corresponding

$T$-modes and solid lines are representing corresponding L-modes.

In Fig. 3, mean value of $\omega^{2}$ about which its value oscillates with variation of wave vector $\boldsymbol{K}$ is larger for array of alternated $\mathrm{Au}$ and $\mathrm{Ag}$ nanospheres than that of alternated $\mathrm{Cu}$ and $\mathrm{Au}$ nanospheres array. Thus array of alternated of $\mathrm{Au}$ and $\mathrm{Ag}$ nanospheres 
array can carry signal of slightly upper energy range than that can be carried by alternated $\mathrm{Cu}$ and $\mathrm{Au}$ nanospheres array. Dislike of array of nanospheres of only one type of metallic nanospheres, in alternated array of nanospheres of different material, there is variation of $\omega^{2}$ at T-mode and L-mode in same direction with the change in wave vector $\boldsymbol{K}$. It helps us to generalize that, group velocity variation at $\mathrm{T}$-mode and $\mathrm{L}$-mode occurs along the same direction with the change in signal frequency $\omega$.

\section{(ii) Group Velocity:}

We have presented group velocity at L-mode only as there is T-mode group velocity is half of the L-mode.
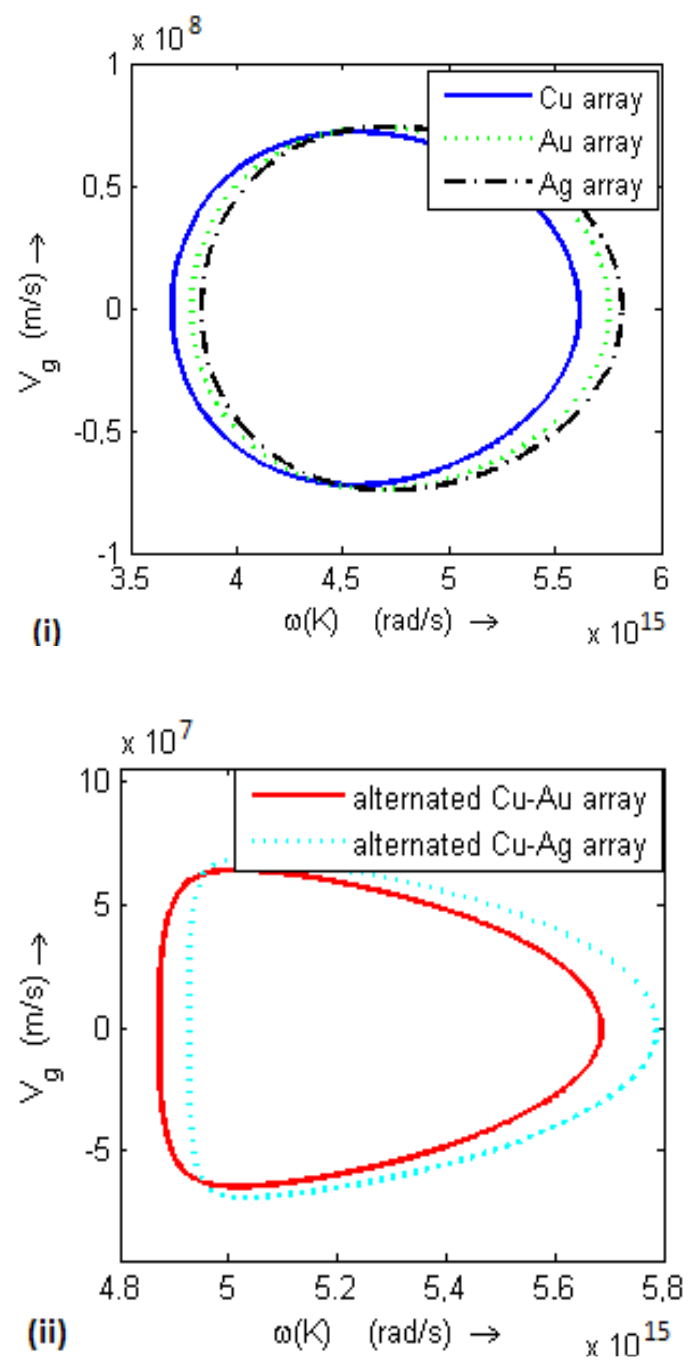

Fig. 4. Group velocity through the array of (i) (a) $\mathrm{Cu}$, denoted by solid line, (b) Au, denoted by dotted line, (c) Ag, denoted by half dotted line and (ii) (a) alternated $\mathrm{Cu}$ and $\mathrm{Au}$, denoted by solid line and (b) alternated Au and Ag, denoted by dotted line, nanospheres.
Fig. 4 shows that there is gradual increase in group velocity and reaches to peak value, corresponds to the central value of $\omega$, resonance oscillation frequency in case of single material nanospheres chain, about which there is variation in value of $\omega$ in dispersion curve, and then again reaches to minimum zero value. There is existence of negative group velocity notifies that signal propagation and phase velocities are exiting in opposite direction. Velocity grows from negative to positive value as there is change in $\omega$ from minimum to mean value of it (resonance oscillation frequency in array of same types of nanospheres). This plot also shows that they have nearly same velocity of signal propagation through array of $\mathrm{Cu}$, array of $\mathrm{Au}$ and array of $\mathrm{Ag}$ nanopsheres as their respective group velocities are $7.18 \times 10^{7} \mathrm{~m} / \mathrm{s}, \quad 7.36 \times 10^{7} \mathrm{~m} / \mathrm{s}$ and $7.45 \times 10^{7} \mathrm{~m} / \mathrm{s}$ respectively in L-mode polarization. We also got their respective values in T-mode; $3.53 \times 10^{7} \mathrm{~m} / \mathrm{s}, 3.62 \times 10^{7} \mathrm{~m} / \mathrm{s}$ and $3.66 \times 10^{7} \mathrm{~m} / \mathrm{s}$ respectively in close agreement with previous carried out work (Brongersma et al. 2000). Furthermore, figure (4) shows that there is decrease in group velocity on using alternated nanospheres array of two different material as $V_{g}$ at L-modes in array of $\mathrm{Cu}-\mathrm{Au}$ is $6.40 \times 10^{7} \mathrm{~m} / \mathrm{s}$ and in array of $\mathrm{Au}-\mathrm{Ag}$ is $6.85 \times$ $10^{7} \mathrm{~m} / \mathrm{s}$. Frequency within which signal can propagate through the array of alternated nanospheres of $\mathrm{Cu}$ and $\mathrm{Au}$ starts earlier than that of alternated array of $\mathrm{Au}$ and $\mathrm{Ag}$ nanosphers. Using the array of alternated nanospheres of $\mathrm{Au}$ and $\mathrm{Ag}$, it is possible to transmit slightly larger volume of data as its frequency range, in which signal can propagate, is slightly larger than array of alternated $\mathrm{Cu}$ and $\mathrm{Au}$ nanospheres.

\section{(iii) Attenuation Coefficient:}

Using the equation (8), we can obtain attenuation coefficient for array of single types of nanospheres material as well as two types of nanospheres material. Attenuations are large at the extreme value of the frequencies and almost constant within these values. This phenomenon also appears in the case of alternated nanospheres array as given in Fig. 5. There is minimum attenuation at the resonance oscillation frequency of individual nanospheres at which there exists maximum group velocity in each chain. Minimum attenuation coefficient for array of nanospheres of $\mathrm{Cu}$ is largest, $0.71 \times 10^{6} \mathrm{~m}^{-1}$, and of $\mathrm{Ag}$ is smallest, $0.53 \times 10^{6} \mathrm{~m}^{-1}$, among 
arrays of $\mathrm{Cu}$, array of $\mathrm{Au}$ and array of $\mathrm{Ag}$ nanospheres. For the array of $\mathrm{Au}$ nanospheres, it is $0.60 \times 10^{6} \mathrm{~m}^{-1}$ at L-mode of polarization. These values, attenuation coefficients, get doubled in T-mode.
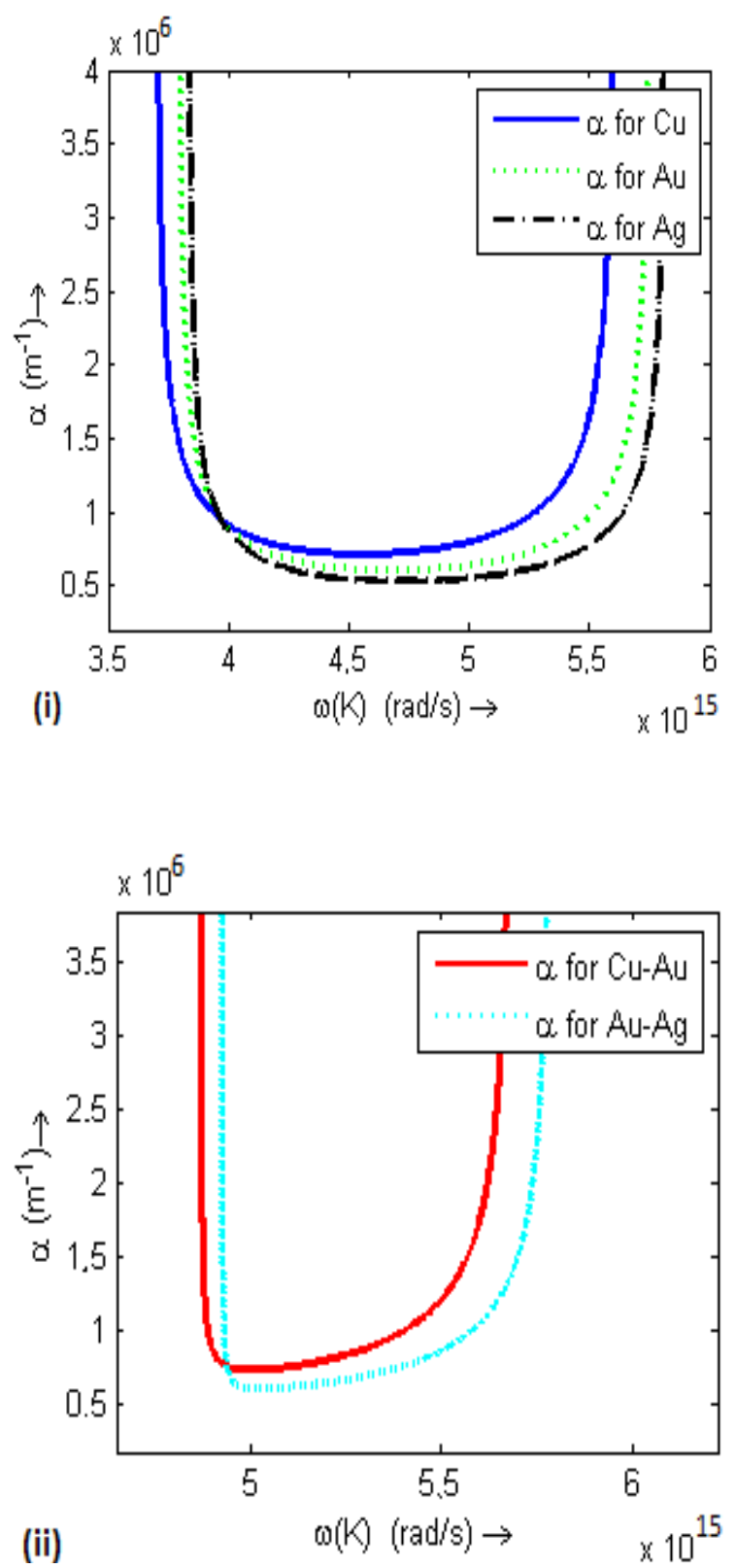

Fig. 5. Attenuation coefficient for the nanospheres array of (i) (a) $\mathrm{Cu}$, denoted by solid line, (b) Au, denoted by dotted line, (c) Ag, denoted by half dotted line and (ii) (a) alternated $C u$ and $A u$, denoted by solid line, (b) alternated $A u$ and Ag, denoted by dotted line.

Minimum attenuation coefficient for array of alternated $\mathrm{Cu}$ and $\mathrm{Au}$ nanoapheres is $0.73 \times$
$10^{6} \mathrm{~m}^{-1}$ and that of alternated nanospheres of $\mathrm{Au}$ and $\mathrm{Ag}$ is $0.60 \times 10^{6} \mathrm{~m}^{-1}$. These values corresponds to attenuation $2 \alpha, 3.17 \mathrm{~dB} / 500 \mathrm{~nm}$ and $2.60 \mathrm{~dB} / 500 \mathrm{~nm}$. From equation (8), it is clearly seen that for small group velocity, there is very large attenuation. On the other hand we have discussed that at L-mode, signal can pass almost two times faster than at T-mode and hence give rise attenuation nearly twice of corresponding Lmode. So it may not be desirable to discuss Tmode than L-mode.

\section{CONCLUSION}

Among the arrays of nanospheres of $\mathrm{Cu}, \mathrm{Au}$ and $\mathrm{Ag}$, we got that group velocities is highest in the array of $\mathrm{Ag}$ nanospheres, $V_{g, 3}=7.45 \times 10^{7} \mathrm{~m} / \mathrm{s}$, and lowest in the array of nanospheres of $\mathrm{Cu}, V_{g, 1}=7.18 \times 10^{7} \mathrm{~m} / \mathrm{s}$. Their corresponding attenuation coefficients are smallest, $\alpha_{L, 1}=$ $0.53 \times 10^{6} \mathrm{~m}^{-1}$ and largest $\alpha_{L, 1}=0.71 \times 10^{6} \mathrm{~m}^{-1}$ among them. Their band width in array of $\mathrm{Ag}$ nanospheres is larger, although by very small value, and hence it is better among those arrays of nanospheres. Among the array of alternated nanaopshers of $\mathrm{Cu}$ and $\mathrm{Au}$, and alternated nanosphers array of $\mathrm{Au}$ and $\mathrm{Ag}$, large bandwidth, small attenuation, and higher group velocity exist for array of alternated nanospheres of $\mathrm{Au}$ and $\mathrm{Ag}$ but not on other. Thus among these two alternated nanospheres array, $\mathrm{Au}$ and $\mathrm{Ag}$ combination is best. In comparison of alternated and non-alternated nanosphers array, array of $\mathrm{Ag}$ nanospheres array is best. Coupling between two different metallic nanospheres is found to be weaker than that exist between same types of metallic nanospheres. Further study can be carried out in the system of alternated

nanosphers hosted in some other metallic substrate can help to increase coupling phenomena (Chen et al. 2011). To obtain relatively higher speed of signal propagation and lower attenuation further research can be carried out by changing the shape of nanostructures used in constructing array (Maier et al. 2002) and host material.

\section{REFERENCES}

Atwater, H. A. 2007. The promise of plasmonics. Scientific America 296: 56.

Barnes, W. L., Dereux, A., and Ebbesen, T. W. 2003. Surface Plasmon subwavelength optics. Nature 424: 824. 
Brongersma, M. L., Hartman, J. W., and Atwater, H. A. 2000. Electromagnetic energy transfer and switching in nanoparticle chain arrays below the diffraction limit. Physical Review B 62: R16356.

Brongersma, M. L., Zia, R. and Schuller, J. A. 2007. Plasmonics-the missing link between nanoelectronics and microphotonics. Applied Physics A 89: 221.

Chen, K. N., Liu, H., Wang, S. M., Zheng, Y. J., Zhu, C., Wang, Y. and Zhu, S. N. 2011. Coherent magnetic plasmon modes in a contacting gold nano-sphere chain on a gold Slab. Optics Express 19: 23782.

Jackson, J. D. 2013. Classical Electrodynamics, Wiley, Delhi.

Johnson, P. B. and Christy, R. W. 1972. Optical constants of the noble metals. Physical Review B 6: 4370.

Kittle, C. 2004. Introduction to Solid State Physics. Wiley, Delhi.

Kreibig, U. and Genzel, L. 1985. Optical absorption of small metallic particles. Surface Science 156: 678.

Kreibig, U., and Zacharias, P. 1970. Surface plasma resonances in small spherical silver and gold particles. ZeitschriftfürPhysik 231: 128.

Maier, S. A., Kik, P. G., Atwater, H. A., Meltzer, S., Requicha, A. A. and Koel, B. E. 2002. Observation of coupled plasmon-polariton modes of plasmon wave guides for electromagnetic energy transport below the diffraction limit, International Symposium on Optical Science and Technology. International Society for Optics and Photonics, pp71.

Ozbay, E. 2006. Plasmonics: merging photonics and electronics at nanoscale dimensions. Science 311: 189.

Quinten, M., Leitner.A., Krenn, J. R. and Aussenegg, F. R. 1998. Electromagnetic energy transport via linear chains of silver nanoparticles. Optics Letters 23: 1331.

Ritchie, R. H. 1957. Plasma losses by fast electrons in thin films. Physical Review 106: 874.

Sattler, K. D. 2016. Handbook of Nanophysics: Nanoparticles and Quantum Dots CRC Press, New York, pp 25-28.

Waser, R. 2012. Nanoelectronics and Information Technology. Wiley-VCH, Germany, pp 769. 